\title{
Lock-in inductive thermography for surface crack detection in non-magnetic metals
}

\author{
by B. Oswald-Tranta*, Ch. Tuschl* \\ * Chair of Automation, University of Leoben, Peter-Tunnerstr.27, 8700 Leoben, Austria, \\ beate.oswald@unileoben.ac.at
}

\begin{abstract}
Inductive thermography is a non-destructive technique for detecting surface cracks in electrically conductive materials. In non-magnetic materials with high electrical and thermal conductivity short heating pulse is necessary, otherwise the thermal signal diminishes too quickly. But if with short heating pulse only little amount of heat is induced in the material then the signal-to-noise ratio (SNR) is too low for reliable defect detection. Applying a sequence of short pulses offers a solution. SNR of the phase image increases with the number of pulses and the cracks become detectable. Aluminum and non-magnetic steel samples with artificial cracks have been manufactured and tested. The influence of the number of pulses, the heating power, the pulse duration, and the crack depth on the SNR is investigated for both materials.
\end{abstract}

\section{Introduction}

In the last years inductive thermography has been well recognized as an excellent non-destructive technique for detection of surface cracks [1-6]. The workpiece to be tested is heated by a short induced eddy current pulse and the surface temperature is recorded by an infrared camera. The heating is generated directly and in a contact-free way below the surface, hence the heating itself does not depend on the emissivity of the surface. Both the eddy current distribution and the heat diffusion are disturbed by surface cracks, therefore by evaluating the infrared images even shallow cracks can be reliably detected [4]. Evaluating the whole infrared image sequence instead of taking into account only one single infrared image, the detectability can be significantly increased and negative effects as inhomogeneous heating or inhomogeneous emissivity values can be strongly reduced [7].

The heated range below the surface is determined by the penetration depth of the eddy currents, which further depends on the excitation frequency and on material properties. Ferro-magnetic materials can be very effectively heated by induced eddy currents due to their high magnetic permeability property. In the case of non-magnetic materials and especially for ones with high electrical and thermal conductivity, as e.g. aluminum, the situation is much more difficult: on the one hand short heating pulse duration is necessary, otherwise the thermal signal diminishes too quickly due to the thermal diffusion. On the other hand with a short heating pulse it is not possible to induce enough heat in the material; therefore the signal-to-noise ratio becomes too low for defect detection. A possibility to overcome this problem is to apply a sequence of short pulses, as it is also done in the lock-in thermography.

Lock-in thermography [8-9] is an often used technique combined with modulated optical heating [9-12] to localize subsurface defects. The technique can be also applied for induction heating by modulating the high-frequency induction current with a lower frequency in order to detect either subsurface defects or surface cracks [12-15].

Applying more pulses requires a longer inspection time and lock-in thermography has only an advantage compared to pulse heating, if the temperature increase generated by one pulse is too low, and the noise is too high. In order to find out, when lock-in thermography has to be really used and how many pulses are necessary, in this paper it is studied how the heating power affects the temperature and phase noise and the signal-to-noise ratio (SNR). It is also investigated, how the noise, the signal contrast and the SNR change with the number of the evaluated pulses. Further it is examined which influence the pulse duration and the crack depth have on these features. Measurement results are presented for aluminium and for stainless steel sample with artificial cracks and also compared with simulation results.

\section{Test samples and experimental setup}

For the experiments two test samples: one aluminium and one stainless steel (AISI 304) sample with the size $30 \times 60 \times 80 \mathrm{~mm}^{3}$ were used. Their material parameters are listed in Table 1. In both samples five artificial cracks were cut along the $8 \mathrm{~mm}$ length with the EDM technique with a depth of $0.5,0.75,1,1.5$ and $2 \mathrm{~mm}$, thus the distance between the cracks is $10 \mathrm{~mm}$. For most of the experiments the samples have been covered with black paint to obtain a higher emissivity.

A $10 \mathrm{~kW}$ induction generator with an excitation frequency of $190 \mathrm{kHz}$ was used. The generator's output current can be controlled in percentage from $10 \%$ up to $100 \%$. The induced heat amount in the sample is approximately proportional to the square of this output signal. The generator was switched on for a given heating pulse duration $\left(t_{p u l s e}\right)$, and after a cooldown period with the same duration the next heating pulse is applied. This sequence is repeated for a number of pulses $\left(N_{\text {pulse }}\right)$. The surface temperature was recorded with an infrared camera containing a cooled InSb detector which is sensitive in the wavelength range $1.5-5 \mu \mathrm{m}$. The camera has a resolution of $320 \times 256$ pixels, and in

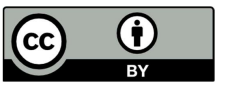


the full frame mode 380 images per second can be recorded. The induction generator and the infrared camera are controlled by a PLC (programmable logic controller) to guarantee a reproducible and synchronized procedure.

Table 1. Material parameters

\begin{tabular}{|c|c|c|c|c|}
\hline Material & $\begin{array}{c}\text { Electrical } \\
\text { conductivity, } \sigma \\
{\left[\Omega^{-1} \mathrm{~m}^{-1}\right]}\end{array}$ & $\begin{array}{c}\text { Thermal conductivity, } \\
\lambda \\
{\left[\mathbf{W ~ m}^{-1} \mathbf{K}^{-1}\right]}\end{array}$ & $\begin{array}{c}\text { Thermal diffusivity, } \\
{\left[\mathrm{m}^{2} \mathrm{~s}^{-1}\right]}\end{array}$ & $\begin{array}{c}\text { Penetration depth } \delta \text {, } \\
\text { (for } f_{\text {exc }}=190 \mathrm{kHz} \text { ) } \\
{[\mathrm{mm}]}\end{array}$ \\
\hline Aluminium & $3.3 \times 10^{7}$ & 218 & $1.2 \times 10^{-4}$ & 0.2 \\
\hline $\begin{array}{c}\text { AISI304 } \\
\text { stainless steel }\end{array}$ & $7 \times 10^{5}$ & 16 & $4 \times 10^{-6}$ & 1.35 \\
\hline
\end{tabular}

\section{Temperature noise at the sound surface}

Experiments with different power, applying from $10 \%$ up to $100 \%$ of the $10 \mathrm{~kW}$ generator, were carried out. The goal was to investigate how the noise and signal behaviour is affected by the temperature increase, which is generated during one single heating period at the sound surface $\left(\Delta T_{\text {sound }}\right)$. For these investigations the back side of the samples without the artificial cracks were used and a region of $31 \times 31$ pixels (appr. $10 \times 10 \mathrm{~mm}^{2}$ ) were in the mid part of the samples selected.

As aluminium has a high electrical conductivity, with one heating pulse of $100 \mathrm{~ms}$ duration only a temperature increase in the range of $\Delta T_{\text {sound }}=0.002-0.15^{\circ} \mathrm{C}$ occurs at the sound surface of the sample. The stainless steel has a lower electrical conductivity and $\Delta T_{\text {sound }}=0.01-0.76^{\circ} \mathrm{C}$ can be achieved varying the power between $10 \%$ and $100 \%$.

The mean temperature of the sample increases steadily by applying more pulses consecutively, see Fig.1a. In order to reduce this effect, for each pulse the starting temperature of the respective pulse is subtracted, see Fig.1b. In both Figs. 1 also the control signal for the generator is included, showing how the pulse heating is switched on and off.

It is to note that usually in lock-in experiments a sinusoid modulation of the heating is used. We have decided for the repeating of square shape pulses, as the results are better comparable with the single pulse measurements.

Different techniques have been suggested [16] for the eliminating the DC part of the heating, one commonly used method is fitting a function $[17,18]$. We used the subtraction of the image at the start of the respective pulse, as this is much quicker, and due to the pulse heating instead of the sinusoid modulation it delivers also appropriate results.

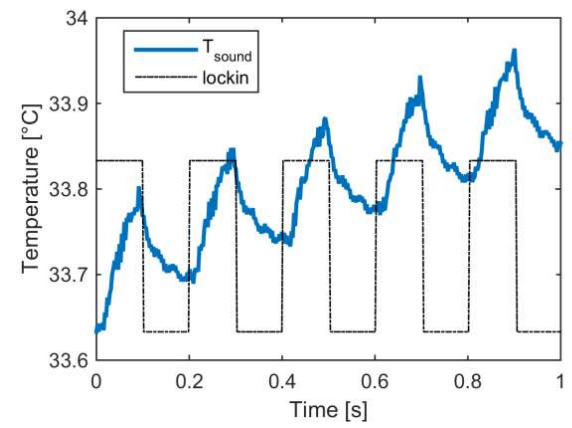

a)

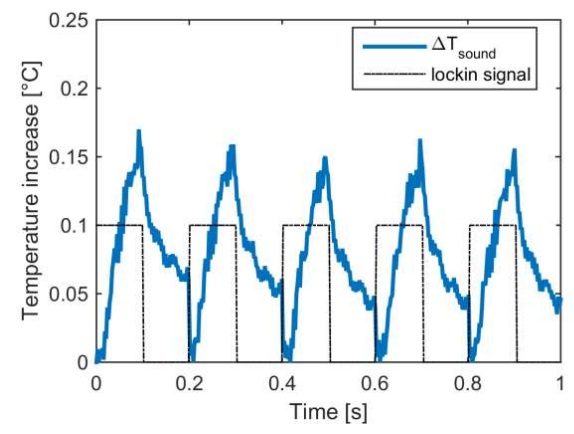

b)

Fig. 1. a: temperature at the sound surface of aluminium during five heating pulses, after applying $100 \%$ power; b: temperature increase $\Delta T_{i}$ obtained by subtracting the starting temperature for each pulse

The noise of the temperature increase is calculated by its standard deviation:

$$
\sigma_{\Delta T}=\sqrt{\frac{\sum_{i \in \text { sound }}\left(\Delta T_{i}(t)-\overline{\Delta T_{i}(t)}\right)^{2}}{n_{p o \text { ints }}-1}}, \text { where } \Delta T_{i}(t)=T_{i}(t)-T_{i}\left(t=t_{\text {pulsestart }}\right)
$$

and $n_{\text {points }}$ denotes the number of pixels at the sound surface taken into consideration for the calculation. A mean value of the temperature increase is calculated by averaging over more pulses. In double logarithmical scale $\sigma_{\Delta T}$ decreases almost as a linear function with the number of pulses, see Fig.2, and the line slope is close to $-1 / 2$. Similar results have been observed by other authors for optically excited lock-in thermography $[8,10,17]$. The temperature noise is mainly independent of the applied heating power, the temperature increase during one heating pulse has no significant influence on the temperature noise, but it is mostly determined by the NETD of the camera [8], which is appr. $20 \mathrm{mK}$ : 


$$
\sigma_{\Delta T}=\frac{N E T D}{\sqrt{N_{p u l s e}}}
$$

It is to note that $\sigma_{\Delta T}$ is the noise of the temperature at the end of the heating pulses. We have chosen to evaluate this temperature noise as this one is representative for the temperature image of a single pulse heating measurement. This noise differs from the noise of the temperature amplitude which is obtained by calculating FFT for more pulses, which is denoted by $\sigma_{A}[8]$ :

$$
\sigma_{A}=\sqrt{\frac{2}{n \cdot N_{p u l s e}}} N E T D=\sqrt{\frac{2}{n}} \cdot \sigma_{\Delta T}
$$

where $n$ denotes the number of images recorded during one period. $\sigma_{A}$ depends on $n$, as it is calculated from all the recorded images during one period. In contrast, $\sigma_{\Delta T}$ is calculated only for the end temperature and therefore it does not depend on $n$ only on $N_{\text {pulse }}$.

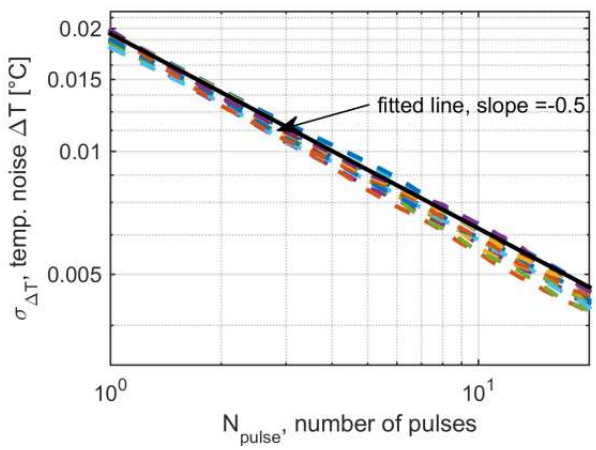

Fig. 2. Temperature noise depending on the number of the pulses plotted for different heating powers for aluminium and stainless steel sample

\section{Phase noise of sound surface}

Instead of evaluating only one single temperature image at the end of the heating pulse, it has been proved $[4,7]$ to be very useful to calculate a phase value by Fourier transformation:

$$
F_{\tau}=\int_{0}^{\tau} T(t) e^{-i 2 \pi / \tau} d t, \quad \Phi=\arctan \left(\frac{\operatorname{Im}\left(F_{\tau}\right)}{\operatorname{Re}\left(F_{\tau}\right)}\right)
$$

where $\tau=t_{\text {pulse }}+t_{\text {cooldown }}$ and $t_{\text {cooldown }}$ is usually taken in the range of $t_{\text {pulse }} / 2$ and $t_{\text {pulse. }}$ In this study, $t_{\text {cooldown }}=t_{\text {pulse }}$ was chosen. The phase image provides an excellent measure for the rate of the heat flow, in which region it increases quicker or slower. It is much less sensitive to disturbing factors such as inhomogeneous heating or inhomogeneous emissivity values. The phase noise is calculated by the standard deviation of the phase values at the sound, crack-free surface:

$$
\sigma_{\Phi}=\sqrt{\frac{\sum_{i \in \text { sound }}\left(\Phi_{i}-\overline{\Phi_{i}}\right)^{2}}{n_{p o \text { int } s}-1}}
$$

This phase noise depends on the heating power and on the temperature increase during one pulse. For the lowest applied power the temperature increase at the sound surface of the aluminium is only about $0.002{ }^{\circ} \mathrm{C}$, which is one order of magnitude less than the temperature noise of about $0.02^{\circ} \mathrm{C}$, therefore the calculated phase is too noisy, as it is mainly calculated for almost random numbers. Applying higher power, the sound temperature becomes also higher; hence the phase noise becomes also less. If the phase is calculated not only for one pulse, but for $N_{\text {pulse }}$ pulses then it decreases with $1 / \sqrt{N_{\text {pulse }}}$ see Fig.3a.

The phase is calculated from all the $n$ images recorded during one heating pulse and cooldown time. Fig. $3 \mathrm{~b}$ shows results where the camera frequency has been set to $100,200,300 \mathrm{~Hz}$, resulting for $t_{\text {pulse }}+t_{\text {cooldown }}=200$ ms in 
$n=20,40$ and 60 , respectively. These results demonstrate that the phase noise decreases with the number images recorded during one period: $\sigma_{\Phi} \sim 1 / \sqrt{n}$.
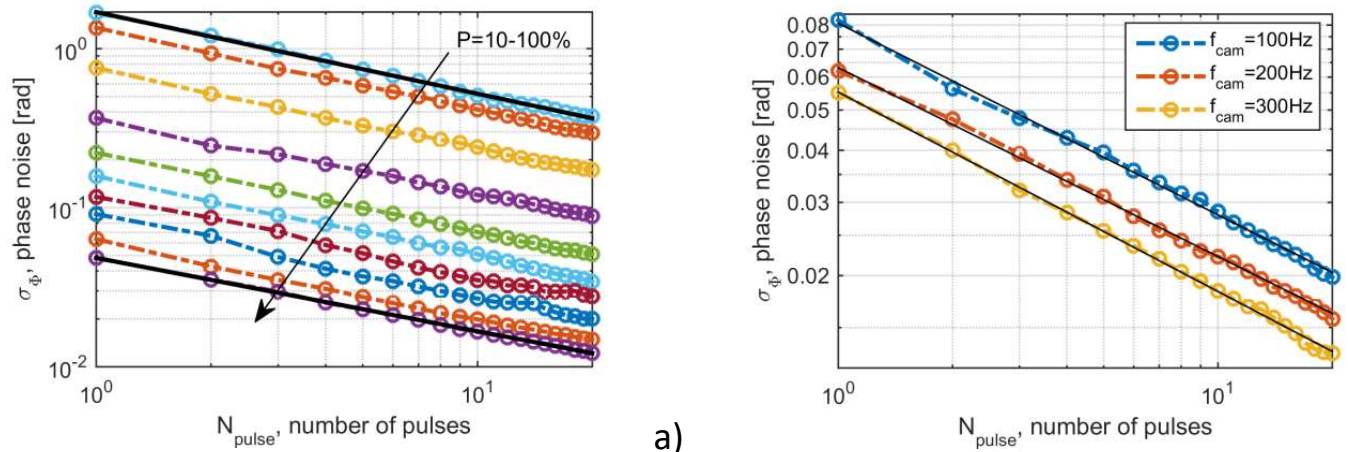

a)

b)

Fig. 3. Phase noise depending on the number of pulses and on the heating power (a) and on the sampling frequency (b), measured for aluminium sample

As it is shown in Fig.3a, the phase noise depends on the heating power, i.e. on the temperature increase during one heating pulse. Fig.4a shows that the phase noise is reciprocally proportional to $\Delta T_{\text {sound }}$ and this relationship does not depend on the material, it is the same for aluminium and for stainless steel. On the other hand, it depends on the sampling rate, that means on the recording frequency of the camera, see Fig. $4 \mathrm{~b}$.

These measurement results are in good agreement with the expected relationship [19]:

$$
\sigma_{\Phi 0} \approx \frac{\sigma_{A}}{\Delta T_{\text {sound }}}
$$

Inserting the Eq.(3), one obtains:

$$
\sigma_{\Phi 0} \approx \sqrt{\frac{2}{n \cdot N_{\text {pulse }}}} \cdot \frac{N E T D}{\Delta T_{\text {sound }}}=\sqrt{\frac{2}{n}} \cdot \frac{\sigma_{\Delta T}}{\Delta T_{\text {sound }}}
$$
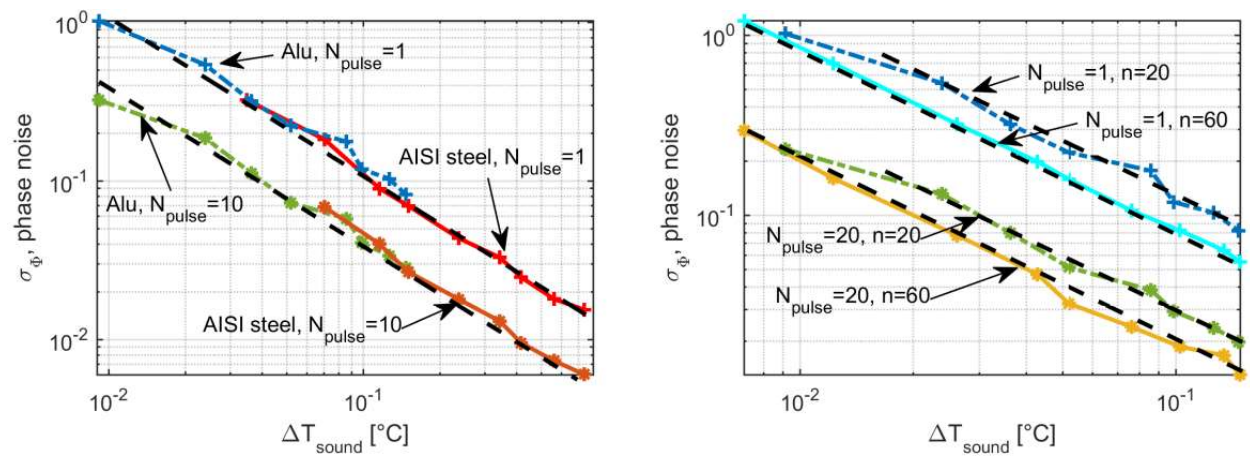

Fig. 4. a: phase noise for aluminium and stainless steel after 1 and 10 pulses depending on $\Delta T_{\text {sound; }} b$ : phase noise depending on $\Delta T_{\text {sound }}$ for aluminium, comparing measurements with $100 \mathrm{~Hz}(n=20)$ and $300 \mathrm{~Hz}(n=60)$ recording frequency.

Fig.5a demonstrates that all the curves of Figs. $4 \mathrm{a}$ and $4 \mathrm{~b}$ lie almost on the same straight line in double logarithmical scale, if $\sigma_{\Phi} \cdot \sqrt{n \cdot N_{\text {pulse }}}$ instead of $\sigma_{\Phi}$ is plotted against $\Delta T_{\text {sound }}$.

Comparing the measured phase noise $\sigma_{\Phi}$ with the temperature noise $\sigma_{\Delta T}$, plotted in Fig.2, then with the corresponding scaling all the phase noise functions are close to the temperature noise lines, see Fig.5b. But in comparison to the theoretically expected value of $\sigma_{\Phi 0}$ a factor of 2.5 had to be introduced:

$$
\sigma_{\Phi} \approx 2.5 \cdot \sigma_{\Phi 0}=2.5 \cdot \sqrt{\frac{2}{n}} \cdot \frac{\sigma_{\Delta T}}{\Delta T_{\text {sound }}}
$$


The factor 2.5 is probably caused by the pulse heating compared to the sinusoidal heating modulation, assumed in the derivation of Eq.(3).

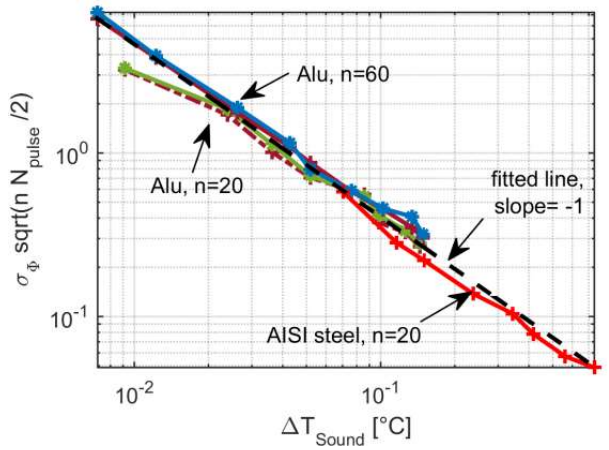

a)

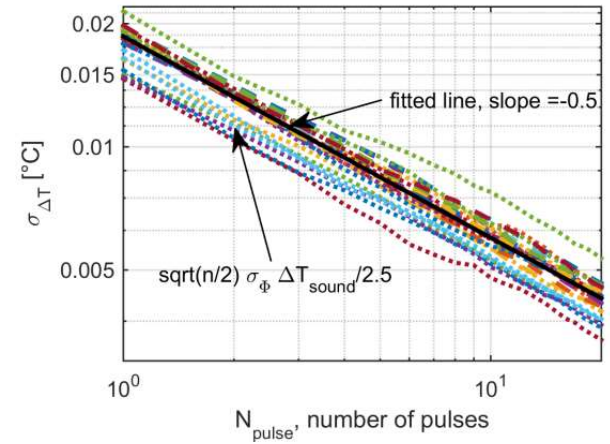

Fig. 5. a: phase noise curves of Figs. $4 a$ and $4 b$ plotted in normalized way; $b$ : normalized phase noise compared to the temperature noise $\sigma_{\Delta T}$

Aluminium has a very low emissivity value, therefore in the previous measurements a black paint has been applied to the sample surface. The inductive heating, in contrary to the optical heating, generates the heat directly in the material and it is not affected by the emissivity. But the emitted radiation is determined by this surface property and hence a black paint may be very useful. Additional measurements have been carried out for the aluminium sample without the black paint. The generated temperature increase due to the induction heating is with and without paint the same: with $100 \%$ heating $\Delta T_{\text {sound }}=150 \mathrm{mK}$. But without the black paint $\Delta T_{\text {sound }}$ seems to be only $8 \mathrm{mK}$, corresponding to $8 / 150=0.053$ emissivity value of the shiny surface. The measured temperature noise of the sample is identical to the curves shown in Fig.2. In order to achieve the same phase noise at the sample without black paint as at the sample with paint $N_{\text {pulse }}=(150 \mathrm{mK} / 8 \mathrm{mK})^{2}=350$ would be necessary.

\section{Signal of a crack}

The temperature contrast is the additional temperature increase at the crack position compared to the sound surface and the relative contrast is its ratio to the temperature increase at the sound surface during the heating pulse:

$$
\begin{gathered}
C_{T}=T_{\text {crack }}\left(t=t_{\text {pulse }}\right)-T_{\text {sound }}\left(t=t_{\text {pulse }}\right) \\
C_{T, R}=\frac{C_{T}}{\Delta T_{\text {sound }}}, \quad \Delta T_{\text {sound }}=T_{\text {sound }}\left(t=t_{\text {pulse }}\right)-T_{\text {sound }}(t=0)
\end{gathered}
$$

The phase contrast is defined as

$$
C_{\Phi}=\Phi_{\text {crack }}-\Phi_{\text {sound }}
$$

It has been shown in previous publications [4], that the temperature and the phase distributions around a vertical crack are determined by two ratios:

- $\quad$ The ratio of the eddy current penetration depth $\delta$ to the crack depth $d$;

- And the ratio of the thermal diffusion length to the crack depth, where the thermal diffusion length $d_{t h}=2 \sqrt{\kappa t}$ describes the distance how far the heat flows in a given time $t$.

If the crack is not vertical, that means its direction is not perpendicular to the surface, then its inclination angle affects also the distributions [4].

If the $\delta<d$, then the temperature and phase value at the crack position is higher than at the sound surface [4]. This is the case e.g. for ferro-magnetic steel, but also for aluminium, if the excitation frequency is $190 \mathrm{kHz}$, see in Tab.1. If the penetration depth is comparable large or larger than the crack depth, then the temperature and the phase is less at the crack than at the sound surface [4], which results in a negative contrast. This is the situation for the stainless steel. Figs. $6 \mathrm{a}$ and $\mathrm{b}$ show both contrasts in dependency on $\Delta T_{\text {sound. }}$. As the heating process is linear, the more heat is applied to the sample, the larger is the temperature increase at the sound surface and at the crack position; and the temperature

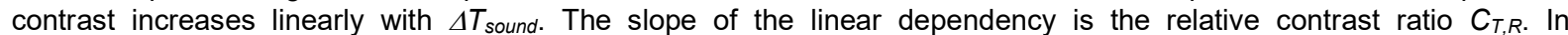
aluminium due to its large electrical conductivity less Joule heating is generated and due to its high thermal conductivity the generated additional heat around the crack is quickly equalized to its vicinity. As the electrical and thermal conductivity of the stainless steel is less, hence the thermal contrast in absolute value is higher, but its value is negative. 
The temperature noise level is mainly independent on the heating power for both materials, as it is also plotted in Fig.6a as confidentiality intervals.

By the calculation of the phase signal in Eq.(4) the dependency on the heating power is eliminated, but the phase noise is influenced by the temperature increase. In Fig.6b the phase contrast is depicted for one pulse, compared to the contrast calculated for 20 pulses. Also the confidentiality intervals with the corresponding phase noise values are shown. For low power and for one pulse the noise is very high, and due to the noise a high phase contrast is shown. With increasing heating power the phase contrast, even for one single pulse, tends to the lower correct value, which is also obtained for 20 pulses. If the temperature increase at the sound aluminium surface is appr. $0.07^{\circ} \mathrm{C}$, then the phase contrast for one pulse has already the same value as after 20 pulses. The noise for one pulse is still high, but by calculating for 20 pulses it becomes significantly lower. If the phase noise is low, either due to the higher heating power or due to applying more pulses, then the phase signal becomes a constant value, independently on the heating power. In aluminium the phase signal has a positive value; and in stainless steel a negative one.
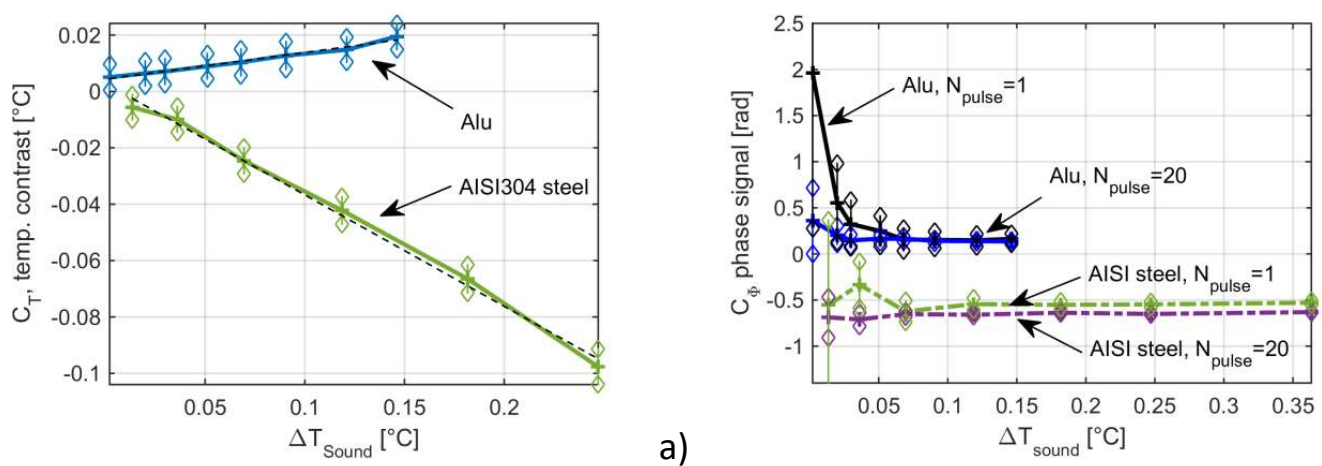

b)

Fig. 6. a: temperature contrast for a $1 \mathrm{~mm}$ deep crack in aluminium and in stainless steel, obtained after 20 pulses; $b$ : phase contrast for the same crack in both materials.

\section{Signal to noise ratio}

Based on the previous equations the temperature and phase signal-to-noise ratio can be expressed as

$$
\begin{aligned}
& S N R_{T}=\frac{C_{T}}{\sigma_{\Delta T}}=\frac{C_{T} \sqrt{N_{\text {pulse }}}}{N E T D}=\frac{C_{T, R} \cdot \Delta T_{\text {sound }} \sqrt{N_{\text {pulse }}}}{N E T D} \\
& S N R_{\Phi}=\frac{C_{\Phi}}{\sigma_{\Phi}}=\frac{C_{\Phi} \cdot \Delta T_{\text {sound }}}{2.5 \cdot N E T D} \sqrt{\frac{n \cdot N_{\text {pulse }}}{2}}
\end{aligned}
$$

Therefore both SNR values increase with $\Delta T_{\text {sound }} \sqrt{N_{\text {pulse }}}$ as it is shown in Figs.7a and $7 \mathrm{~b}$.

For the detectability of a crack the SNR should have at least a value of 4 . So it is an important question, how large temperature increase during one period is necessary, in order to achieve this SNR value. Or, if using a given induction coil setup and only a certain temperature increase could be generated, how many pulses have to be taken into consideration for an SNR $=4$.

For aluminium with the setup used in these experiments only with the highest power $\left(\Delta T_{\text {sound }}=0.15^{\circ} \mathrm{C}\right)$ and at least after 20 pulses $S N R_{T}=4$ could be achieved, see Fig.7a. The situation is much better for the phase, with the same power even with one pulse $S N R_{\Phi}=4$, see Fig.7b. Applying 20 pulses, then a temperature increase of $0.05^{\circ} \mathrm{C}$ is also enough $(\mathrm{P}=60 \%)$ for the same $S N R_{\phi}$. For stainless steel $S N R_{T}=4$ after 20 pulses, if $\Delta T_{\text {sound }}=0.05^{\circ} \mathrm{C}$. For the phase the same $\Delta T_{\text {sound }}=0.05^{\circ} \mathrm{C}$ is enough even after one pulse to achieve $S N R_{\Phi}=4$, or if 20 pulses are evaluated, then $\Delta T_{\text {sound }}=0.02^{\circ} \mathrm{C}$ is also enough for the same SNR.

The ratio of the two SNR values is given by

$$
\frac{S N R_{\Phi}}{S N R_{T}}=\frac{1}{2.5} \sqrt{\frac{n}{2}} \cdot \frac{C_{\Phi}}{C_{T, R}}
$$

As $C_{T, R}$ about double, and $C_{\phi}$ about four times so high for the stainless steel than for aluminium, therefore the ratio of the SNRs is also about double so high for the steel. The ratio of the SNRs is about 2 for aluminium and about 4 for stainless steel, if $n=60$. 


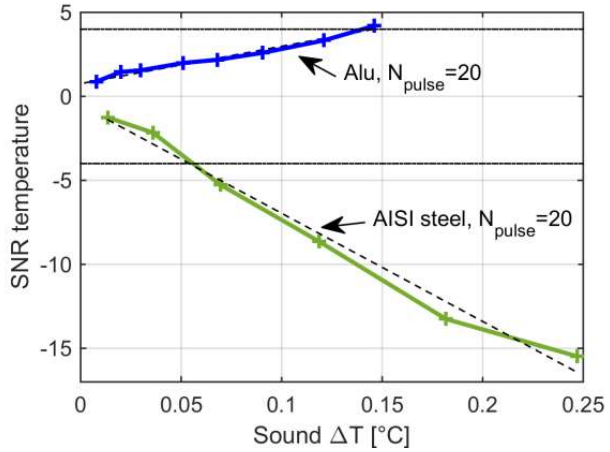

Fig. 7. a: SNR for temperature (a) and for phase a)

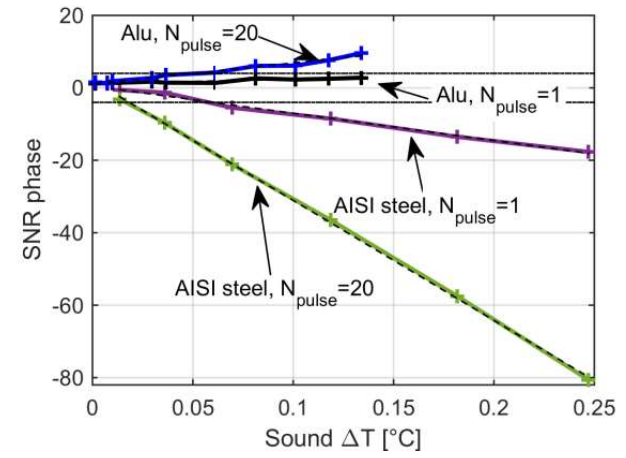

b)

Fig. 8 shows the temperature and phase image after 20 pulses received with $P=100 \%$ for aluminium (Figs. $8 a$ and $8 \mathrm{c}$ ) and with $\mathrm{P}=50 \%$ for stainless steel (Figs.8b and $8 \mathrm{~d}$ ). In both materials $\Delta T_{\text {sound }}$ is similar and has the value $0.15-0.18^{\circ} \mathrm{C}$. The crack depths are $2,1.5,1,0.75$ and $0.5 \mathrm{~mm}$ from the top to bottom, respectively. In the middle of the images the red line marks the position, where the profiles over the crack with $d=1 \mathrm{~mm}$ were evaluated in the previous results. The red rectangle shows the region, used for the calculation of the sound noise of the SNR values. In aluminium as well in the temperature as in the phase image the cracks are visible due to higher values, and in the stainless steel due to lower ones. In the temperature image the cracks are much less visible than in the phase image, as the SNR is lower. But also another problem can be noticed: the heating of the samples is not homogeneous, both sides close to the coil windings have a higher temperature, causing a very inhomogeneous temperature image. On the other hand, in the phase image this effect is well suppressed and the image delivers an almost constant signal along the whole sample.
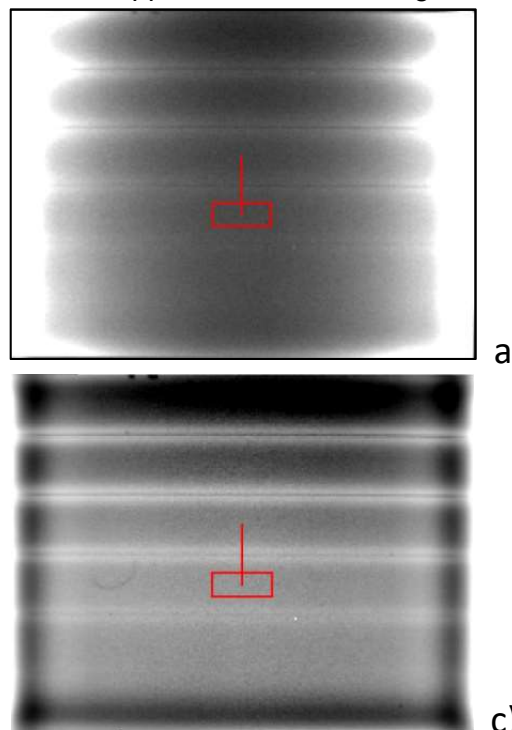

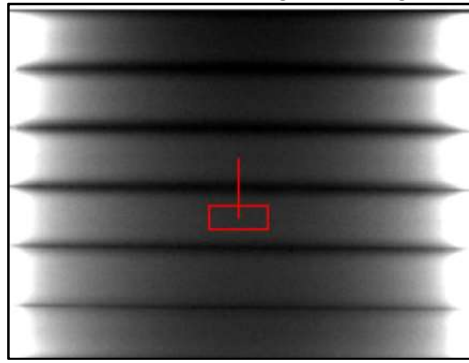

b)

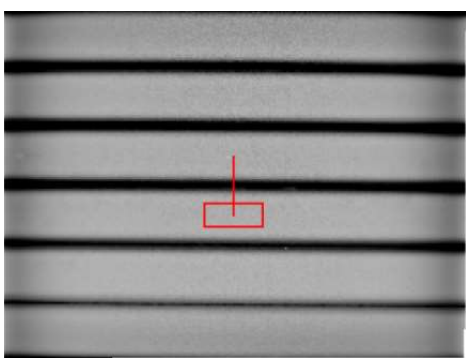

d)

Fig. 8. Temperature (a) and phase (c) image for aluminium and temperature (b) and phase (d) image for stainless steel, obtained after 20 pulses, each with 100 ms heating duration.

\section{Different crack depths}

The signal of a crack depends on its depth, as the eddy current and the heat diffusion are stronger deviated by a deeper crack. As it is shown in Fig.9, the deeper the crack, the larger is the resulting contrast. The shallowest crack with $0.5 \mathrm{~mm}$ has a very weak signal in aluminium and could not be detected. This is caused partially by the geometrical form of the artificial crack with a width of $0.3 \mathrm{~mm}$, and it has more the shape of a shallow mould, which is very different from a real crack.

In Fig.10 the phase SNRs are depicted for the same cracks. It is to see, that a deeper crack causes a larger signal, and its SNR reaches with less number of pulses an SNR limit, which is enough for reliable detection. 


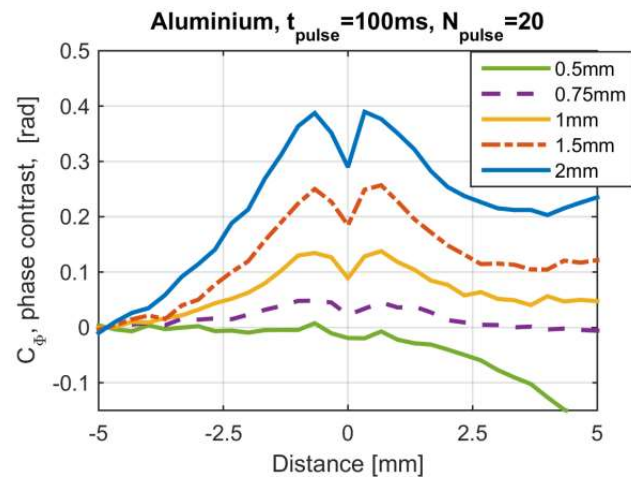

a)

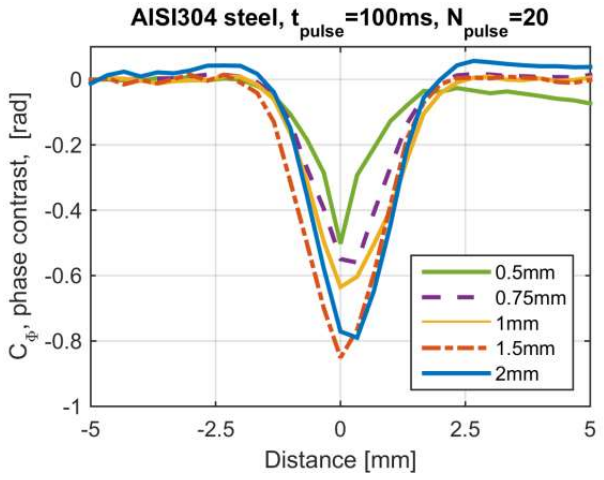

b)

Fig. 9. Phase contrast of the artificial cracks in aluminium (a) and stainless steel (b) obtained after 20 pulses
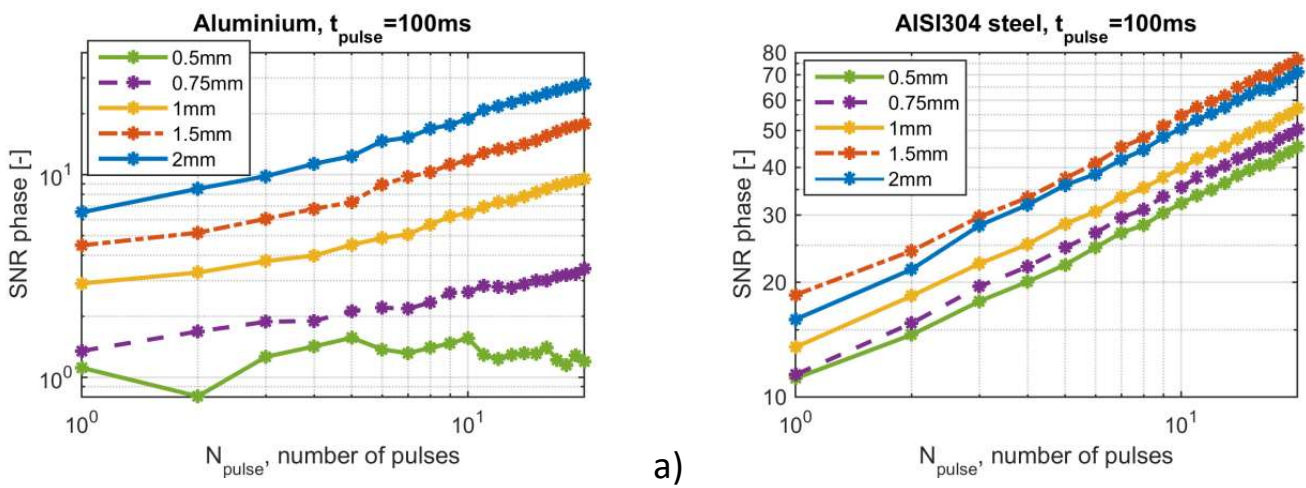

a)

Fig. 10. Phase SNR of the artificial cracks in aluminium (a) and stainless steel (b).

b)

\section{Influence of pulse duration}

It has been shown in the previous sections that a higher heating power increases the SNR. The question occurs, whether this higher heating could also be achieved with longer heating pulse duration. If the heating is longer, then the temperature due to the heat diffusion equalizes during the heating pulse, which reduces the signal. This process is even more significant for materials with high thermal conductivity, as e.g. aluminum.

Experiments were carried out with different pulse lengths in the range of $50-300 \mathrm{~ms}$ to investigate this effect. The phase noise decreases with longer heating duration. This has two reasons: a longer heating pulse causes higher temperature increase, which reduces the phase noise and on the other hand longer heating pulse is recorded with more images, which additionally reduces the phase noise. But the phase contrast itself depends on the pulse duration. Fig.11 shows the measured results and Fig.12 the simulated ones [15,20]. For aluminium both results are in good agreement, showing that the phase contrast has a maximum in the range of $100-150$ ms heating pulse, but the measured phase values are slightly less than the simulated ones. For stainless steel there is more difference between both results, but it is to recognize that with longer heating pulse the phase contrast in absolute value decreases.
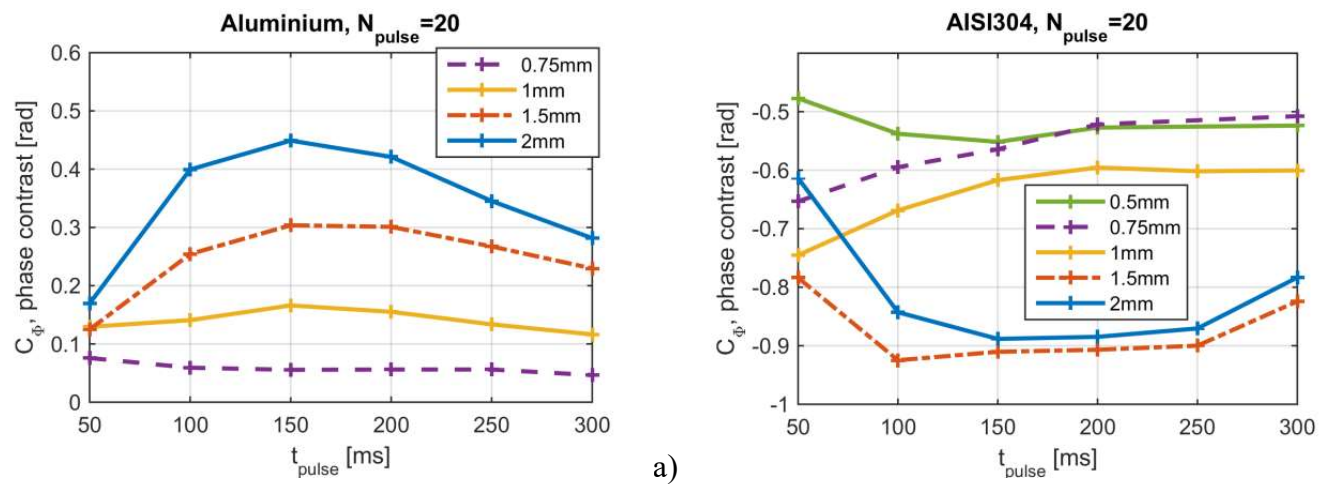

b)

Fig. 11. Phase contrast for different crack depths in aluminium (a) and in stainless steel (b) depending on the heating pulse duration 

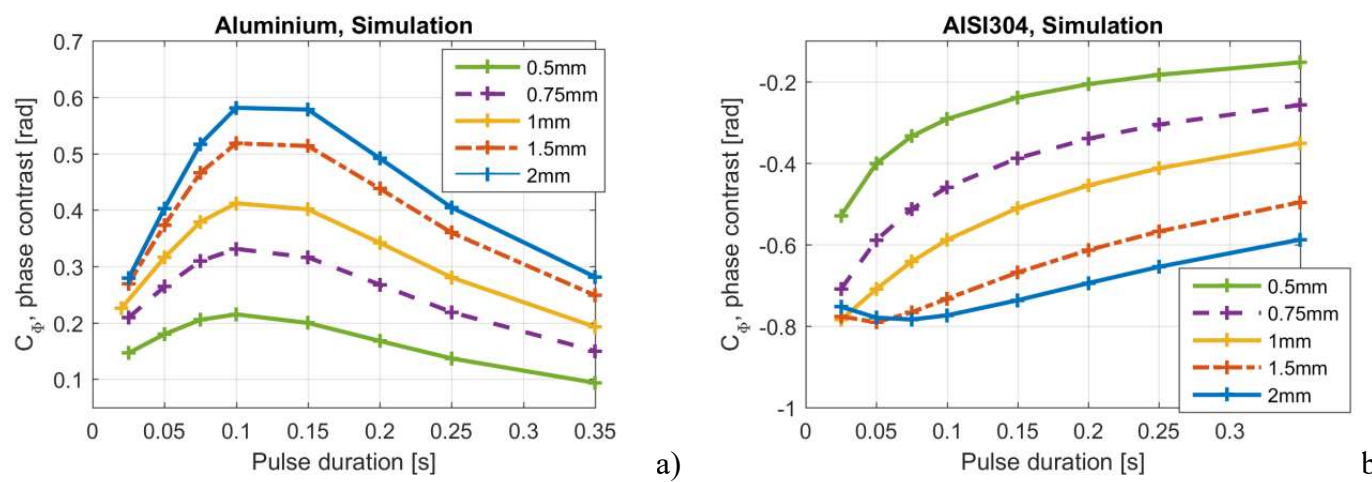

b)

Fig. 12. Simulated phase contrast for different crack depths in aluminium (a) and in stainless steel (b) depending on the heating pulse duration

In Fig.13 two phase images are compared, obtained with the shortest (50ms) and with longest (300ms) pulses in aluminium. The cracks by using $300 \mathrm{~ms}$ pulses have about the same phase contrast or even lower as by using the short pulses, according to Fig.12a. The noise is lower and the $S N R_{\Phi}$ is higher for the $300 \mathrm{~ms}$ pulse, but the image of the $50 \mathrm{~ms}$ pulses is sharper. Due to the shorter time the width of the signal is less, causing a sharper image. This means further that for the detectability of signals additionally to the contrast and to the SNR also its sharpness, which means also the gradient value, should be taken into consideration. This has been already suggested by Balageas et al. for flash thermography experiments for detecting subsurface defects [21].
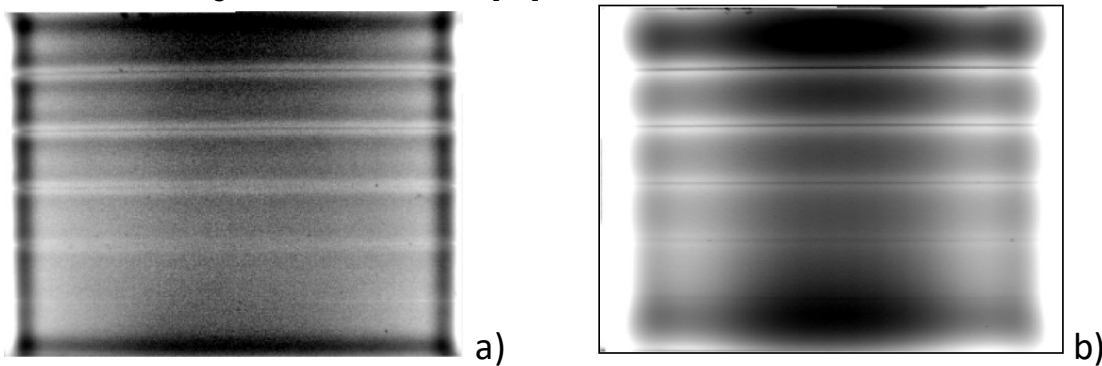

Fig. 13. a: temperature image with 50 ms pulse heating (80 pulses); b: phase image with 300 ms pulse heating (20 pulses).

\section{Ferro-magnetic sample}

In ferro-magnetic samples the induction heating is much more efficient due to the high magnetic permeability of the material. But also in this case applying multiple pulses can be very useful, if the temperature increase generated during one pulse is too low. Fig.14 shows an example, which has a crack about $12 \mathrm{~mm}$ long and $1 \mathrm{~mm}$ deep. In the region around the crack the surface has been ground, creating here a shiny surface with low emissivity. Assuming an emissivity value of $1, \Delta T_{\text {sound }}$ would be $0.065^{\circ} \mathrm{C}$ at the ground surface and $0.13^{\circ} \mathrm{C}$ elsewhere, which means that the grinding has reduced the emissivity value to the half of its original value. The phase noise at the ground surface after one single pulse $\sigma_{\Phi}=0.136 \mathrm{rad}$, which will be reduced by calculating the phase image for 20 pulses to $\sigma_{\Phi}=0.136 / \sqrt{20}=0.03 \mathrm{rad}$ and the crack can be well detected even at the ground, shiny surface.
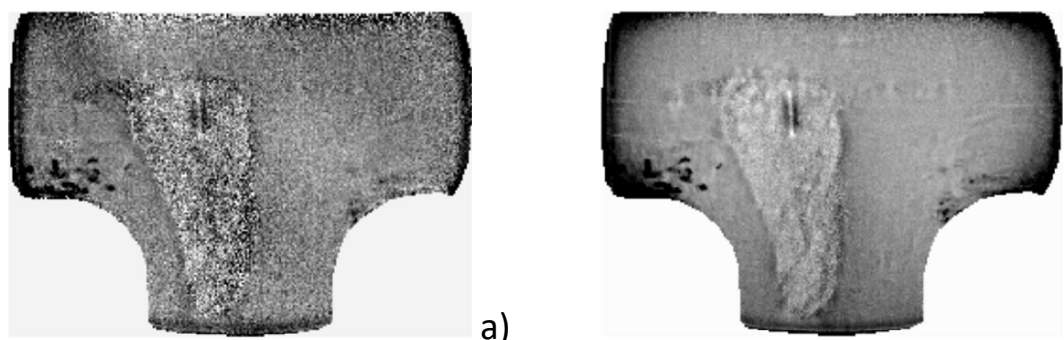

b)

Fig. 14. a: phase image after one single $100 \mathrm{~ms}$ heating pulse of a ferro-magnetic sample with low heating power; $b$ : the phase image calculated for 20 pulses of the same sample 


\section{Summary and conclusion}

Inductive thermography is an excellent non-destructive testing method to detect surface cracks in metallic materials. But there are cases, when a single pulse does not generate enough heat in the material. This can be the situation, when the coupling of the induction heating is not sufficient, e.g. using a large induction coil for a small sample, or if a non-magnetic material has a high electrical and thermal conductivity, as aluminum. In these situations applying consecutive pulses may be a solution.

It has been investigated how the heating power, the temperature increase generated during one heating pulse and the number of pulses influence the temperature and the phase signals. These results allow an estimation how many pulses are necessary for a given heating power to achieve e.g. an SNR=4 value. It has been also shown, that the SNR of the phase is significantly larger than the one of the temperature.

Applying different pulse lengths shows, that the phase contrast has a maximum around 150 ms pulse length in aluminium and also in stainless steel shorter pulses give better results. If the heating pulse is longer, then due to the heat diffusion the signal is quicker equalized and the contrast decreases.

Investigation of the signal dependency on the crack depths proves that the deeper the crack, the larger is the contrast, which gives a good possibility to estimate the crack depth from the inductive thermography results.

\section{References}

[1] U.Netzelmann, G.Walle, S.Lugin, A.Ehlen, S.Bessert, B.Valeske, "Induction thermography: principle, applications and first steps towards standardisation", QIRT Journal 13(2), 170-181, (2016)

[2] J.Wilson, G.Tian, I.Mukriz, D.Almond, "PEC Thermography for Imaging Multiple Cracks from Rolling Contact Fatigue", NDT \& E International, 44(6), 505-512, (2011)

[3] J.Vrana, M.Goldammer, J.Baumann, M.Rothenfusser, W.Arnold, "Mechanism and models for crack detection with induction thermography", Proc.AIP Conf. , 975, (2008)

[4] B.Oswald-Tranta, "Induction Thermography for Surface Crack Detection and Depth Determination", Appl. Sci.8, 257; http://dx.doi.org/10.3390/app8020257 (2018)

[5] Ch.Srajbr, "Induction Excited Thermography in Industrial Applications", in Proc. 19th WCNDT, Munich, (2016)

[6] DIN 54183:2018-02, "Non-Destructive Testing-Thermographic Testing- Eddy-Current Excited Thermography", available online: https://www.din.de (2018)

[7] B. Oswald-Tranta, "Time-resolved evaluation of inductive pulse heating measurements," QIRT Journal 6(1), 319, http://dx.doi.org/10.3166/qirt.6.3-19 (2009)

[8] O.Breitenstein, M.Langenkamp, "Lock-in Thermography", Springer Series in Advanced microelectronics, (2003)

[9] D.Wu and G.Busse, "Lock-in thermography for nondestructive evaluation of materials," Revue Générale de Thermique, vol. 37, no. 8, pp. 693-703, (1998).

[10] Ch.Maierhofer, Ph Myrach, R.Krankenhagen, M.Röllig, H. Steinfurth,"Detection and Characterization of Defects in Isotropic and Anisotropic Structures Using Lockin Thermography", J. Imaging, 1, 220-248; doi:10.3390/jimaging1010220 (2015)

[11] C.Meola, G.M.Carlomagno, A.Squillace, A.Vitiello, "Non-destructive evaluation of aerospace materials with lockin thermography", Engineering Failure Analysis 13 380-388 (2006)

[12] T.Sakagami, S.Kubo, "Applications of pulse heating thermography and lock-in thermography to quantitative nondestructive evaluations", Infrared Physics \& Technology 43 211-218 (2002)

[13] G.Riegert, T.Zweschper, and G.Busse, "Lockin thermography with eddy current excitation", QIRT Journal 1(1), 21-31 (2004).

[14] U.Netzelmann, G.,Walle, "Induction Thermography as a Tool for Reliable Detection of Surface Defects in Forged Components", in Proc. of the $17^{\text {th }}$ World Conference on Non Destructive Testing, Shanghai, China, 2528 October (2008).

[15] B. Oswald-Tranta, "Lock-in inductive thermography for surface crack detection", in Proc. Thermosense XL, Orlando, 2018.

[16] Gupta R, Breitenstein O. Unsteady-state lock-in thermography-applications to shunts in solar cells. QIRT J 2007;4(1):85-105.

[17] C.Antolis, N.Rajic, "Optical Lock-in Thermography for Structural Health Monitoring -A Study into Infrared Detector Performance", 6th Asia Pacific Workshop on Structural Health Monitoring, (2016).

[18] S.Pickering, D.Almond, "Matched excitation energy comparison of the pulse and lock-in thermography NDE techniques", NDT\&E International 41 (2008) 501-509

[19] M.Goldammer, private communication, (2018).

[20] ANSYS, Inc., [Online]. Available: http://www.ansys.com

[21] D.L. Balageas, J.-M. Roche, F.-H. Leroy, "Comparison and ranking procedure for an objective assessment of thermographic NDE methods", in Proc. 13th QIRT conf. Gdansk, Poland, (2016). 